\title{
ASSESSMENT OF GESTATIONAL DIABETES MELLITUS RELATED STRESS USING A SPECIFIC SCALE ADMINISTERED AS AN ONLINE MOBILE APPLICATION
}

\author{
A. SRINIVASAN, D. KRISHNARAJAN, P. SARANYA*, T. G. JITHIN, SNEHA THOMAS, V. VARADHARAJAN
}

Department of pharmacy practice, JKKMMRF College of pharmacy-Erode, Namkkal District, Tamilnadu, India

Email: saranyasathiapal23@gmail.com

Received: 24 Feb 2019 Revised and Accepted: 23 Apr 2019

\section{ABSTRACT}

Objective: Aim of the study was to assess the Gestational Diabetes Mellitus (GDM) related stress among pregnant women using an online mobile application based specific stress scale for GDM.

Methods: This was a prospective observational study. All GDM patients who have used the Gestational Diabetes Stress Scale (GDSS)-mobile application within the study period were included (176 patients). Their total and subscale stress scores were analyzed.

Results: This study found that $52.84 \%$ of the total population needed clinical attention for GDM related stress. The subscale scores revealed that $65.91 \%$ of the population needed clinical attention for emotional burden, $15.34 \%$ of the population needed clinical attention for medication-related stress, $69.89 \%$ of the population needed clinical attention for social or economical stress and $36.36 \%$ of the population needed clinical attention for health care set up related stress.

Conclusion: Based upon this study we conclude that GDSS is a good invention. There existed a gap in measuring GDM related stress in pregnant women and GDSS is a solution for the same.

Keywords: Gestational diabetes mellitus, Gestational diabetes-related stress, Gestational diabetes stress scale

(C) 2019 The Authors. Published by Innovare Academic Sciences Pvt Ltd. This is an open-access article under the CC BY license (http://creativecommons.org/licenses/by/4.0/) DOI: http://dx.doi.org/10.22159/ijpps.2019v11i6.32783

\section{INTRODUCTION}

Gestational Diabetes Mellitus (GDM) is a condition of any degree of glucose intolerance with onset or first detected during pregnancy. Prevalence of GDM increasing worldwide (21 million new cases per year) [1]. According to International diabetes federation, $16 \%$ of the children born alive in 2013 had complications due to hyperglycemia Goals for Glycemic Control in Pregnancy are like, Fasting 60-90 $\mathrm{mg} / \mathrm{dl}$, Pre meal $<100 \mathrm{mg} / \mathrm{dl}, 1 \mathrm{hr}$ postprandial $<140 \mathrm{mg} / \mathrm{dl}, 2 \mathrm{~h}$ postprandial $<120 \mathrm{mg} / \mathrm{dl}$, Bedtime $<120 \mathrm{mg} / \mathrm{dl}$, and 2:00-6:00 A. M. $60-90 \mathrm{mg} / \mathrm{dl}$ [2]. GDM can cause complications in both mother and foetus. Major Fetal complications are Macrosomia, spontaneous abortion, congenital malformation and intrauterine death and maternal complications and risk of diabetes recurrence in future pregnancies, the future possibility of diabetes mellitus, polyhydramnios, pregnancy toxemia, urinary tract infection, candidiasis, higher incidence of premature childbirth and caesarean delivery[3]. Studies found that prevalence of diabetes is high among females [4] and GDM can further increase the risk for the same.

GDM will increase emotional disequilibrium during pregnancy. It is well documented that pregnancy is linked with unique psychological stress and diabetes also linked with significant distress. In addition to the stress of pregnancy, GDM is also a stress factor [5-7]. Women with GDM also experience shock, fear and stress $[8,9]$. Maternal stress during pregnancy can cause emotional or cognitive problems in childlike attention-deficit/hyperactivity, anxiety and language delay [10].

Stress can affect GDM control by influencing proper diet compliance, medication adherence or glucose monitoring. To prepare a suitable plan for GDM related stress proper identification of the stress level along with the possible sources for the same is needed. Manikandan et al. in Tamilnadu concluded from their study that providing proper patient counseling services and creating awareness regarding the condition can improve patient's medication adherence [11]

Primary objective of the study was to assess the GDM related stress in pregnant women through the administration of an online mobile application based specific scale for gestational diabetes mellitus.

\section{MATERIALS AND METHODS}

Study Design: This was a prospective observational study.

Study duration: Six months

Study population: 176 pregnant women with GDM

Data collection: Data collection was done through GDSS mobile application.

\section{Study criteria}

All pregnant women with GDM irrespective of gestational weeks, naturally pregnant women and In vitro fertilization (IVF) pregnant women with GDM were included in the study. Non-consenters and incomplete data entries were excluded from the study.

\section{Materials used}

A mobile application called 'GESTATIONAL DIABETES STRESS SCALE' It is available in Google play store (https://play.google.com/ store/apps/details?id=com.byzero.gdss)

\section{RESULTS AND DISCUSSION}

Age wise distribution

Among the total study population majority of the patients were from 24 to 29.12 y which is $44.88 \%$ of the population, followed by 30 to 34.12 years (25\%), 18 to 23.12 years (16.48) and the least were from the age group of greater than or equal to $30 \mathrm{y}(13.67 \%)$.

\section{Educational status}

Most of the patients in the study population were graduate which is $43.75 \%$ of the study population followed by postgraduate $(21.59 \%)$, metric $(18.18 \%)$, higher secondary $(12.05 \%)$ and less number of the population was illiterate (3.98\%).

\section{Occupation status}

Among the study population, $51.13 \%$ were homemakers, $14.78 \%$ were self-employees, $5.68 \%$ were IT professional, $4.54 \%$ were from 
bank sectors, $3.40 \%$ were from government sectors and $20.45 \%$ were from other categories.

\section{Monthly family income}

Majority of the study population (40.04\%) were from less than or equal to $10,000 \mathrm{Rs}$, followed by 10000 to less than or equal to 20000 (32.96\%), 20000 to less than or equal to 30000 (11.93\%), greater than or equal to $40000(10.22 \%)$ and the least was from 30000 to less than or equal to 40000 (4.54\%).

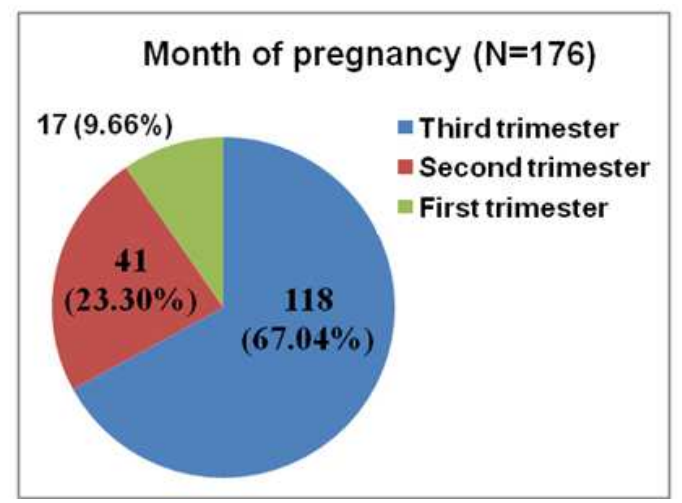

Fig. 1: Month of pregnancy

\section{Month of pregnancy}

Highest number of population $118(67.04 \%)$ of the study population were in the third trimester during the study period, $41(23.30 \%)$ of the population were in the second trimester and the least of the population, 17 (9.66\%) were in the first trimester.

\section{Pregnancy sequence}

Majority of the population were conceiving for the first time which is $50.56 \%$, followed by second time $39.77 \%$, fourth time $(3.40 \%)$, fifth time $(2.27 \%), 1.70 \%$ of the population were conceiving for the sixth or seventh time and one patient was conceiving for the eighth time.

\section{History of GDM in last pregnancy}

Among total study population 87 patients were conceiving for more than one time and among that 30 patients had a history of GDM in their last pregnancy which is $34.49 \%$ of the study population and 57 patients did not have a history of GDM in their last pregnancy.

\section{Family history}

Among the study population, 97 patients in the study population had a family history of diabetes which is $55.11 \%$ of the population.

\section{GDM management}

Majority of the patients needed only diet and lifestyle modifications which is $53.98 \%$. $25.57 \%$ of the study population was on insulin, $14.20 \%$ patients were on oral hypoglycemic agents and least part of the population $6.25 \%$ were on both insulin and OHA.

Table 1: GDM management

\begin{tabular}{lll}
\hline GDM management & Number & Percentage (\%) \\
\hline Both insulin and OHA & 11 & 6.25 \\
Diet, exercise and Lifestyle modification & 95 & 53.98 \\
Insulin only & 45 & 25.57 \\
OHA & 25 & 14.20 \\
\hline
\end{tabular}

\section{Awareness monitoring}

Monitoring awareness of GDM among pregnant women was one of the objectives of the study. A questionnaire containing nine questions was used to monitor the awareness of pregnant women with GDM. Patient's responses to each question were as follows.

\section{Knowledge about GDM}

The question asked was 'Do you know what is meant by gestational diabetes mellitus?' And Majority of the study population $56.81 \%$ replied they know what GDM is and $43.19 \%$ of the population replied they don't know what GDM is.

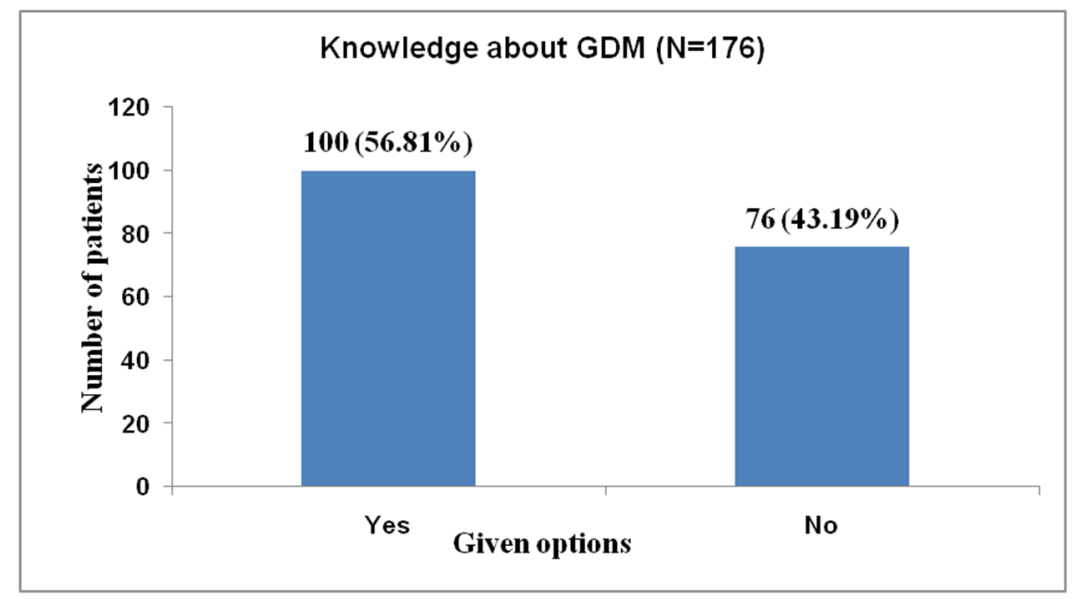

Fig. 2: Knowledge about GDM

\section{Occurrence of GDM}

The question was 'GDM can occur only during the first pregnancy, do you agree?'. A large number of the population $83(47.15 \%)$ responded 'No' which means they don't agree with the given statement. $27(15.35 \%)$ patients believed GDM can occur only during the first pregnancy and $66(37.50 \%)$ patients chose don't know' option for the question. 


\section{GDM will disappear after delivery or not}

The question was 'GDM will disappear after delivery. Do you agree?'. A good proportion of the population of 109 (61.13\%) patients believed GDM will disappear after delivery. 15 (8.52\%) patients thought GDM will not disappear after delivery and 52(29.55\%) patients replied 'don't know' to the question.

\section{Risk of an unborn child}

'Unborn child is at risk if the mother is untreated. Do you agree?' This was the fourth question and the highest number of population $127(72.16 \%)$ responded like an unborn child is at risk if the mother is untreated. $16(9.06 \%)$ patients felt unborn child is not at risk if the mother is untreated and $33(18.75 \%)$ patients response was they don't know the answer.

\section{Risk for future type 2 diabetes}

The question asked was 'women with GDM are at an increased risk of future type 2 diabetes. Do you agree? Patient responses were noted. The given statement was agreed by $98(55.69 \%)$ patients and $23(13.06 \%)$ of patients felt women with GDM are not at an increased risk of future type 2 diabetes and 55(31.25\%) patients response was they don't know the answer.

Table 2: Risk factors of GDM

\begin{tabular}{ll}
\hline Which of the following are the risk factors of GDM? & Number of patients \\
\hline Family history DM & 112 \\
Pre-pregnancy obesity & 94 \\
GDM in a previous pregnancy & 83 \\
Any/All of the above & 76 \\
Don't know & 46 \\
\hline
\end{tabular}

\section{Risk factors of GDM}

The patients were given five options for the question of what are the risk factors for GDM. The patient response was as follows:

Table 2 shows, patients had a choice to choose more than one option. 112 patients believed the family history of diabetes is the risk for GDM, 94 patients thought pre-pregnancy obesity as the risk factor, 83 patients thought GDM in last pregnancy as the risk factor, 76 patients thought any or all of these options are the risk factors and 46 patients were didn't know what are the risk factors for GDM

\section{Management of GDM}

The question was 'What are the treatment options for GDM?' Three options along with all of the above and don't know were given and the patient responses were noted. For this question, the patient selected more than one option. 141 patients thought lifestyle modification was the management option for GDM, 92 patients thought oral hypoglycemic agents, 80 patients thought insulin injection, 72 patients selected all of the above and 20 patients selected they don't know the answer.

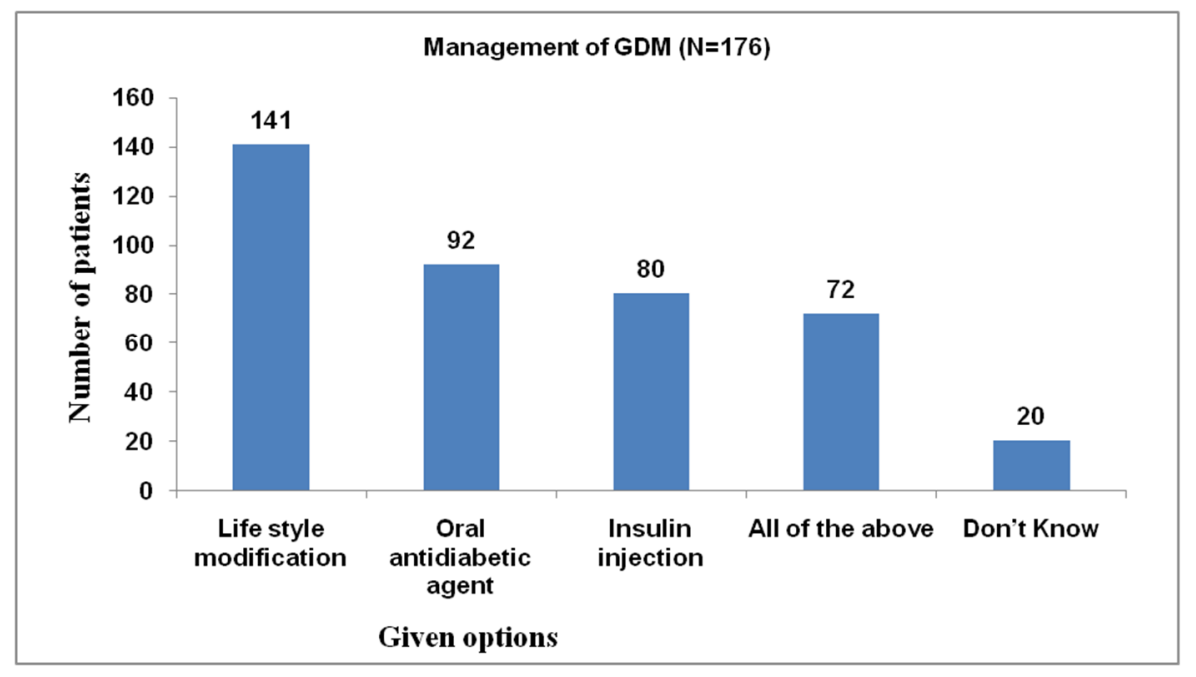

Fig. 3: Management of GDM

\section{Suitable time period for diagnosis}

The question asked was 'Which of the following time period is appropriate for the diagnosis of GDM?'; 40 patients thought 12 to 16 $\mathrm{w}$ are the appropriate time period for GDM diagnosis, 38 patients believed 18 to $22 \mathrm{w}$ are the appropriate time, 98 patients thought 24 to $28 \mathrm{w}$ are the suitable time, 21 patients thought any time period during pregnancy is suitable to diagnose GDM and 63 patients didn't know what the suitable time period to diagnose GDM is.

\section{Tests used to detect GDM}

'Which of the following test is used to detect GDM?' was the last question in the awareness questionnaire. 51 patients thought urine test was suitable to detect GDM, 102 patients thought blood test, 96 patients thought blood test following glucose load, 40 patients felt any or all of the above and 17 patients selected don't know an option for this question.

\section{Gestational diabetes-related to stress}

Gestational Diabetes Stress Scale (GDSS) was used to detect the stress levels in pregnant women. This is a 16 item scale which gives the total score to monitor the overall stress level and four subscale score to detect the type of distress.

\section{Total GDSS score}

Total GDSS score gives an overall stress level in patients. Whether the patient needed clinical attention or not could be detected using total GDSS score. Among the study population, $52.84 \%$ of the study population needed clinical attention and $47.16 \%$ of the population did not need clinical attention. 


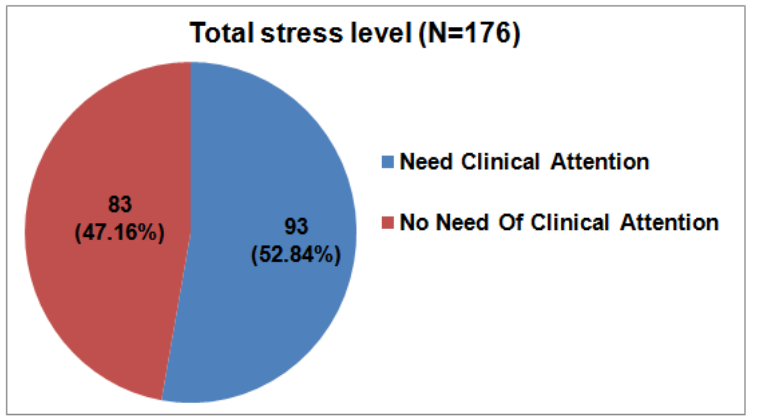

Fig. 4: Total GDSS score

\section{Emotional burden}

This is the first sub scale of the GDSS. Which contain 7 items, the 7 items included in this subscale are related to the emotional state of the patient like 'I felt sad when diagnosed with GDM','I lost interest or pleasure in doing things after diagnosed with GDM',' I feel scared and sad that even though GDM will disappear after delivery, in future I may get diabetes', 'I feel my behavioral pattern has changed after GDM diagnosis', 'I am afraid that because of GDM, I may end up in cesarean', 'I feel decreased productivity in my works' and 'I feel depressed that my child may also get affected because of my GDM'. Among the total study population $65.91 \%$ of the population needed clinical attention and $34.09 \%$ of the population did not need clinical attention.

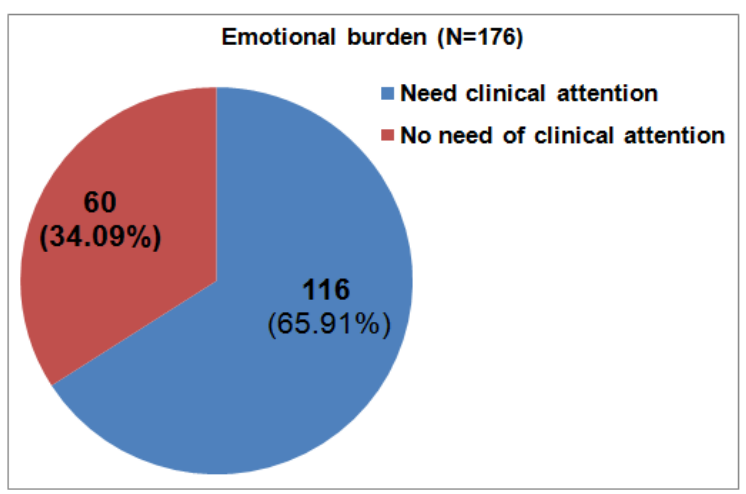

Fig. 5: Emotional burden

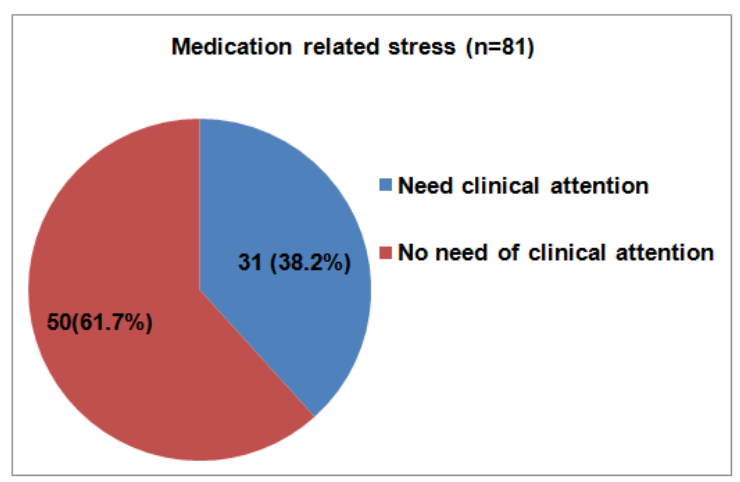

Fig. 6: Medication-related stress

\section{Medication-related stress}

This is the second subscale in GDSS, which include 3 items. Patients only on lifestyle modification were not monitored for medicationrelated stress. The 3 items included in this subscale are related to medication. Which are like 'Taking insulin for GDM is painful', 'Every time when I take insulin or oral anti-diabetic agents I feel bad' and 'I feel that I am taking too much of medications'. Among the 81 patients who were on medication for GDM, $38.2 \%$ of the study populations needed clinical attention and $61.7 \%$ of the study populations did not need clinical attention.

\section{Social or economical stress}

This is the third subscale of the GDSS which include 3 items. The three items included in this subscale are 'I spend more money than what I estimated because of GDM', 'I don't get enough support from my family and friends' and 'I am not able to be normal because of various restrictions due to GDM.'. $69.89 \%$ of the populations needed clinical attention for social or economical stress and $30.11 \%$ of the populations did not need clinical attention for the same.

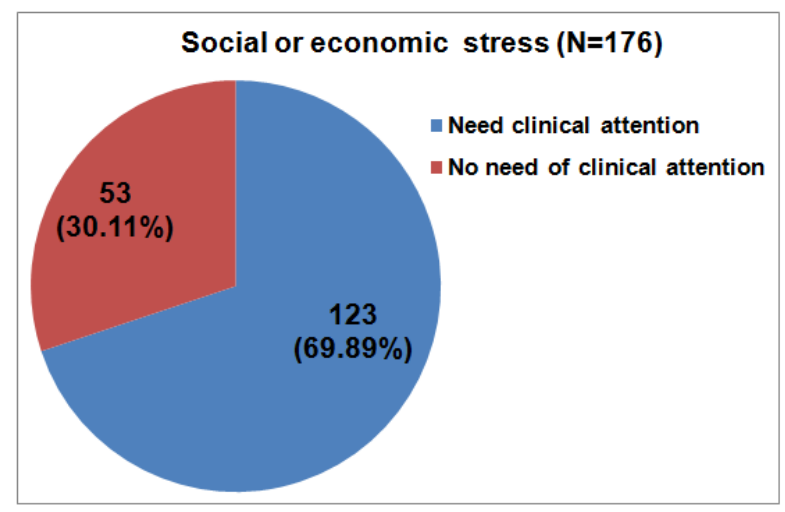

Fig. 7: Social or economical stress

\section{Health care set up related stress}

This is the fourth subscale of GDSS which contains 3 items. The three items included in this sub scale are 'I don't get enough time with the physician to discuss my issues', 'I am not aware about my condition and also the precautions that I should take' and 'I feel no one is bothered for my GDM and I don't get proper care as I required'. In the total study population $36.36 \%$ needed clinical attention and $63.64 \%$ did not need clinical attention for health care set up related stress.

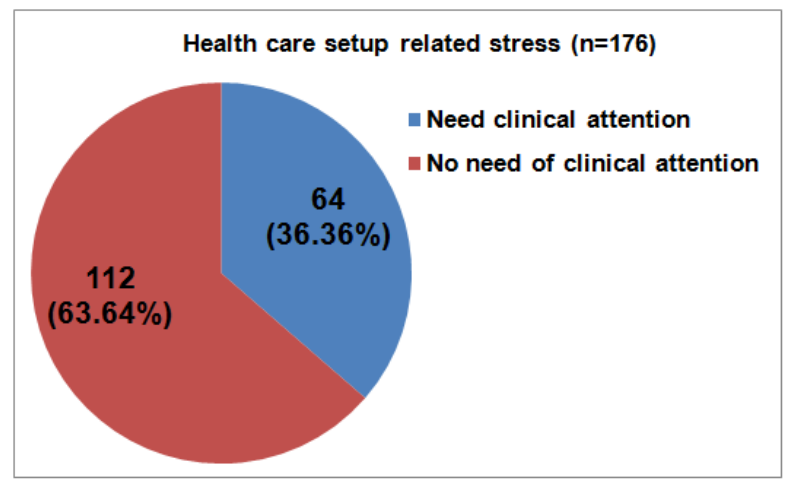

Fig. 8: Health care set up related stress

No specific stress scale was available to monitor stress level in pregnant women with GDM before the introduction of GDSS. However, there are studies conducted to detect GDM related stress in pregnant women using more than one stress scales. Lydon $\mathrm{k}$ et al. conducted a similar study. They investigated the psychological stress associated with diabetes during pregnancy (a pilot study). Examined the psychosocial profile of 25 women with gestational diabetes mellitus and compared them to 25 non-diabetic pregnant 
women. They used the pregnancy experience scale (PES), the depression, anxiety stress scale (DASS), the problem areas in diabetes scale (PAID-5) and Perceived social support scale (PSSS). The GDM group reported a significantly greater ratio of pregnancy 'hassles' to pregnancy 'uplifts'. The GDM groups also had a significantly higher depression score and were twice as likely to score above the cut-off for possible depression. Elevated levels of diabetes-related distress were found in $40 \%$ of women with GDM. In addition, the GDM group reported less social support from outside family. Thus the study indicates the need of psychological screening in GDM and the provision of psychological support in some cases.

Limitations of this work were that it was conducted among women who knew how to use a mobile application and also among women who could understand English. We cannot check the GDM related stress among Illiterate women.

\section{CONCLUSION}

This study indicated $52.84 \%$ of the total population needed clinical attention for GDM related stress which is a significant amount and proper precaution must be given to stress management while preparing an action plan for GDM management. Analyzing subscale score reveals $65.91 \%$ of the population needed clinical attention for the emotional burden,it indicates the patient's need for an emotional support, $69.89 \%$ of the population needed clinical attention for social or economic stress, $15.34 \%$ of the population needed clinical attention for medication-related stress and $36.36 \%$ of the population needed clinical attention for health care set up related stress. Comparing data of the stress level and awareness monitoring clearly indicates that there is a lack of proper awareness among GDM patients and they need clarity about the medical condition, management options, drugs etc. Lack of proper awareness itself can increase the stress level in patients. Pharmacists can solve this issue by providing proper awareness through proper patient counseling services.

\section{AUTHORS CONTRIBUTIONS}

All the authors have contributed equally

\section{ACKNOWLEDGMENT}

Nil

\section{CONFLICT OF INTERESTS}

Declared none

\section{REFERENCES}

1. Khan R, Ali K, Khan Z. Maternal and fetal outcomes of gestational diabetes mellitus. Goma J Med Sci 2013;11:88-91.

2. Montserrat Balsells. Glibenclamide, metformin, and insulin for the treatment of gestational diabetes: a systematic review and meta-analysis. Br Med J 2015;350:h102.

3. Letícia Nascimento Medeiros Bortolon, Luciana de Paula Leao Triz, Bruna de Souza Faustino, Larissa Bianca Cunha de Sa Denise Rosso Tenorio Wanderley Rocha, Alberto krayyem arbex-gestational diabetes mellitus: new diagnostic criteria. Open J Endocrine Metabolic Diseases 2016;6:13-9.

4. Shrinivas Sangappa Tamboli, Ramamurthy B. Prevalence and predictors of diabetes among adults in rural Dharwad, India: a cross-sectional study. Asian J Pharm Clin Res 2018;11:94-9.

5. Hayase M, Shimada M, Seki H. Sleep quality and stress in women with pregnancy-induced hypertension and gestational diabetes mellitus. Women Birth 2014;27:190-5.

6. Spirito A, Ruggiero L, Bowen A, Mcgarvey S, Bond A, Coustan D. Stress, coping, and social support as mediators of the emotional status of women with gestational diabetes. Psychology Health 1991;5:111-20.

7. Lawson EJ, Raja ram S. A transformed pregnancy: the psychological consequences of gestational diabetes. Sociol Health Illness 1994;16:536-62.

8. Kim C, Vahratian A. Self-rated health and health care use among women with histories of gestational diabetes mellitus. Diabetes Care 2010;33:41-2.

9. Morrison MK, Lowe JM, Collins CE. Australian women's experiences of living with gestational diabetes. Women Birth 2014;27:52-7.

10. Nicole M Talge, Charles Neal, Vivette Glover. Antenatal maternal stress and long-term effects on child neurodevelopment: how and why. J Child Psychol Psychiatry 2007;48:245-61.

11. Manikandan R Nandamuri, Sri Sai Sudha, Logaraj M. Assessment of medication adherence among patients with hypertension and diabetes mellitus in urban field practice. Asian J Pharm Clin Res 2018;11:130-2. 